\title{
INTEGRATION OF THE VISUAL ELEMENTS OF ART AND PERSONALITY FACTORS IN PROCESS OF CHARACTER DESIGN
}

\author{
Nada Miketić (D), Ivan Pinćjer (D), Ana Lilić (1) \\ University of Novi Sad, Faculty of Technical Sciences, \\ Department of Graphic Engineering and Design, Novi Sad, Serbia
}

\begin{abstract}
Video game design is an extensive field of study and complex creation process which synthesize various scientific areas such as information technologies, art and design, psychology, and other social sciences. During the time, character design evolved into a separate field of study. Character design process is constantly in front of new challenges, due to the changes in the digital era, and a considerable quantity of information that people are exposed to. The most attention is put on the visual appearance of the character during the creation process. There is a large number of influential factors on the visual representation of the character, some of them are: game genre, the target audience, platform. However, in the design stage, excellent knowledge of the visual elements of the art is required because they have the most influence on the visual representation of the character. Analogous to the real world and the real human characters, virtual characters have "personality" as well. In order to accent the specificity of the character, not only the art elements have to be considered, but personality characteristics. The paper will provide an overview of the elements of art and personality factors such as archetype, facial expression, view, body posture. The purpose of this paper is to emphasize the mutual connection of the art elements and personality factors and their final influence on the unique appearance of the character.
\end{abstract}

Key words: character design, the elements of art, virtual characters

\section{INTRODUCTION}

Character design for video games and movies have always been the most challenging process for creative industry workers. This paper explains design elements for "good" character visual and background knowledge that is essential for the creation of an iconic character. In the search for a deeper explanation of the character design process, the process itself will be analysed from two different aspects. The first is related to the creative process of thinking in which character is conceived. This aspect comprehends a psychological approach to the character, which involves the term archetype that has an important role in the process of creating a new character. The second aspect concern the importance of basic elements of art, their practical use, and symbolism behind.

\section{2. "BIRTH" OF THE CHARACTER}

The first step in the process of character creation is doubtless the idea. Thus there is an idea for the particular character design, therefore a preliminary idea about the character nature. This initial idea about the character can be related to either their personality traits, their background story or both. The character is always in the service of the story (Tillman, 2011a). The basic principles of the character design are grounded in profound knowledge of archetypes. Thanks to archetypes people can recognize patterns that assemble personality and character traits, at first encounter with a particular character. Regardless the used medium - storytelling, more, television or video games - character design is based on the use of the universal categories which observer remember and based on these categories observer can easily identify new characters. According to this, the character design process, the use of archetypes as a guideline can enable the precise visual appearance of the character.

\section{ARCHETYPES}

There is a variety of archetypes that are established by Swiss psychologist and psychoanalytic Carl Gustav Jung. However, there will be analysed only relevant ones for this paper - ones that apply the storytelling and entertainment industry. Archetypes mentioned above are: hero, shadow (villain), fool, anima/animus, mentor, and trickster. 
Follows are presented these archetypes and their substantial traits, the ones that observer can immediately notice in the first encounter with the particular archetype. This also can serve as a directive during the character design process - the goal is to identify traits which are the most expressed at particular archetype, characteristics that are universally perceived as the same. If it is required to illustrate character which represents a hero then will be useful to become known with heroes structural elements. The archetype of the hero is defined throughout the following traits: very brave, unselfish and eager to help regardless of the consequences.

- The archetype of the villain, according to Jung, is defined as a shadow. The shadow character is closely related to animal instincts. He or she is described as ruthless, mysterious, unkind, unpleasant and evil.

- The archetype of the fool is present in the story in order to test the main character. The fool is optimistic, innocent and full of luck. The character that is represented as a fool makes unpredictable situations and in that way complicates relationships and make the story more interesting.

- $\quad$ Archetype animus is the female fellow, partner to the male character, whereas animus represents a male partner to the female character. This character has a role to pease female and male drives and provides interest and possibility for the love in the sorry.

- The archetype of the mentor, which is present more in the movies than in video games (mentor in video games is commonly used to walkthrough player through tutorials). Mentor represents intellect and stability for the protagonist, wisdom, and support. He is wise and owns an enormous quantity of knowledge and has a mission to lead hero on his way heading to great doings. A mentor is often illustrated as an older male or female, because of the connection between old age and wisdom.

- The last archetype is the trickster. Trickster can be bowed both evil and good side of the story. Regardless of the supported side, trickster tends to turn the story for their own benefit.

Knowing the specific traits of the particular archetype is not enough for good design of the character. There is also required the knowledge of the basic elements of art and the way of using them in order to describe character traits of the specific archetype (Tillman, 2011b).

\section{THE VISUAL APPEARANCE OF THE CHARACTER}

\subsection{The facial expression}

The face is one of the most important channels of communication among humans. Facial expressions can reveal emotions which character is feeling, face reveals the identity of the character and across the face, the character can be "red". Accordingly, the face is the inevitable part which has to be analysed and paid special attention to it during the process of the character design.

In the communication process, the character can raise or lower brows, to frown, shrink or spread eyes and mouth. Although movement of the face cam express particular emotions and define character, movement of the face can also emphasize the importance of the conversation or spoken words. For instance, raised brows to highlight an importance of something or can be understood as a sign to pay attention to something. Knowing various facial expressions and what they mean is necessary in order to present identity of the character that is essential for identification with the character. Since it is a large number of facial expressions, it is virtually impossible to distinguish all their meanings. During the design process of an animated character commonly used facial expressions and emotions which are the same as the majority of people and can be recognized easily (Isbister, 2006a). There are four basic emotions (Figure 1):

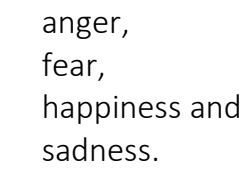

Since these emotions are common for all, they can be easily illustrated, because each of enlisted emotion is expressed the same to by all characters. It is certain that there is a "recipe" for illustration of basic emotions because they are usually expressed in the same way by the majority of the characters.

It is required that the facial expressions and emotions of the fictional character be as much as possible realistically presented - whether the character is very stylised of character very detailed and realistic - 
because the aim of animated movie and video game is that the person consumes content as much as possible and to identify themselves with the character, a to induce emotional reaction to person (Isbister, 2006b).

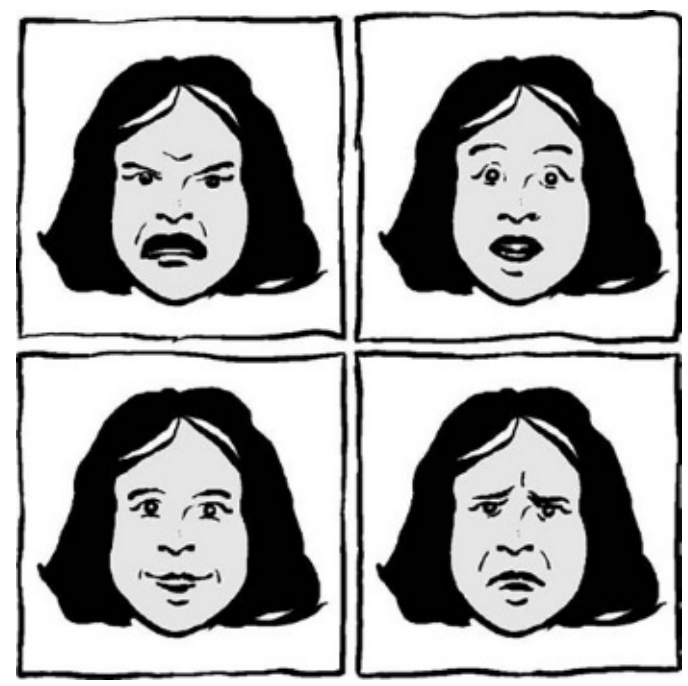

Figure 1: Four basic emotions (Isbister, 2006)

\subsection{The look}

An inevitable part of the appearance of the character is the look. Similarly, with the face, the look has also a great indicator of the personality traits of the character and their emotions. The look can imply what character is interested in (if looking around environment), in that way person who is in the interaction with the character is paying attention to the same thing as the virtual character, regardless it is movie or video game character. Furthermore, the look can indicate whether the character is interesting or not. Importance of the look and eyes in the character design process can easily be conveyed with eye colour of additional effects for instance presence of magic in the eyes in form of smoke or fire (Isbister, 2006c).

\subsection{Posture and shape of the body}

As mentioned earlier, the same idea applies to the posture and shape of the body when identifying the character. Posture, the position of the body and movement (for animated characters), convey large quantity if the information related to characters personality, emotions and overall personality traits. For example, if it is required to design the hero character, it is common that construction of the body of the hero is muscular, athlete posture, nice - looking overall appearance and harmonious proportions of the body. There is no coincidence in the design of the heroes' body. Enlisted common characteristic of the appearance of the hero have to describe their ability for instance strength, fast movements can fight an opponent and easily overcome physical obstacles. On the other hand, villains can also be muscular, but their constitution of the body is different than heroes. Villains usually have a less harmonic body or less regular proportions than the hero character. They are often presented as extremely skinny or fat or muscular. Also, generally known common body positions which reveal the certain emotional state of the character and attention are: lowered shoulders (for the sad or disappointed character), spread hands and legs with slightly bended knees usually represent combat posture and attention to attacking. Knowing body language and position that character takes in particular situations, is essential for good character design (Isbister, 2006c).

\section{IMPORTANCE OF THE BASIC ELEMENTS OF ART IN THE PROCESS OF CHARACTER DESIGN}

Basic elements of art are in service of clear presentation the most characteristic traits of the particular character. They emphasize the archetype of the character. Basic elements of art can be described as the mirror of the personality traits of the character. 


\subsection{Line}

The line is the simplest basic element of the art. Not only is it used to define the shape of the character, but also leads observers eye. It makes connections between shapes and communicates the direction of the body lines and movement. The first and most noticeable use of the line in the character design is outlined which defines the shape of the character. Regardless of the visual style in which character is presented, characteristic of the lines which enable visual representation of the character traits are line thickness, orientation, position and type (solid or dashed). Further, in the text, there is an explanation of each characteristic of the line and how they affect the visual appearance of the character.

- $\quad$ Line thickness - in essence, the line does not have thickness. It is a one - dimensional object. However, for the people to easily conceive lines, there has to be thickness. Different line thickness is differently perceived by the people. For instance, the thin line has loos effect, it is barely noticeable and soft. Whereas thick lines convey a completely opposite impression. When designing a character, if creating an honest, pure and serious character, the use of thin lines would be an appropriate choice. Opposite of that, when using thick lines the character appearance will be perceived as rough, heavy, strong and solid.

- Line orientation - this characteristic has also much influence on the presentation of the character traits. Horizontal lines can convey the sense of calmness, strength, and stability, whereas the use of vertical lines is the most appropriate for well balanced, self - confident and renowned character. Diagonal lines, as well as curved lines, suggest dynamic, movement in rising or fall.

- The position of the line - can refer to a contour line or structure line (the lines that define details of the character). Additionally, the position of the line also defines the relationship between elements of the character.

- $\quad$ Type of the line - straight lines as often perceived as stable and consistent, curved lines are dynamic, energized and natural (Solan, 2015a).

From this explanation of the different line characteristics, it is clear that the combination of the characteristics of the line and ways of using them determine the personality traits of the character.

\subsection{Shape}

The hope is in the close connection with the line, it cannot be detached from the line. The shape can be used for defining countries and details of the character. The most commonly used shapes in the character designing process are a circle, triangle, and square. A key technique in process of character design is to create a character from the basic shapes. Basic shapes enable easy understanding and recognition of the character by the observer. Interpretation of the basic shapes differs from each other for particular shapes. Circle - connotation of the circle are connected with the symbolism of its shape, which is common for the majority of people and cultures. Those are kindness, unity, peace, and whole. If the character consists mainly of circular shapes, the character will be in most cases perceived as positive, benevolent, merciful, well balanced, natural and fond of care for the community. Consequently, circular shapes are most commonly used in character design for children, younger audience or heroes with a high level of stylization. Triangle - dependence of its use, there are a variety of understandings of its symbolism. If the triangle is oriented with its peak on the top, it is usually described as stable, strong and restful shape. On the other hand, if the triangular shape is oriented with peek directing down than it symbolizes misbalance, instability, and sense of tension. Triangle can be analysed from two different perspectives: energy and temperament. Energy can mean dynamic, movement and speed, whereas temperament represents peoples character traits that can be described with a triangle shape. Those are aggression, hostility, dishonesty, passion, and sexuality. Square - square shapes and rectangular shapes are most commonly perceived as strong and the most stable shapes. Besides these understandings, rectangular and square shapes are often understood as certain, rational, strong, make and pure. Therefore, they are mainly used for the design of the hero character (Solan, 2015a).

\subsection{Silhouette}

This element of the art is the most responsible for creating the recognizable appearance of the character (Figure 2). Observing the character through silhouette enable the analogy of the line and shape at the same time. This technique is the most popular in the process of character design in the most renowned studios in the creative industry. Besides it represent a great way of the basic construction of the character, it is also 
relevant for the reconstruction of the character, in the analysis of the existing characters. The most important feature that can be easily conveyed using silhouettes is character recognition. Silhouette can be analysed from two different aspects. First is static appearance which has to provide answers on the questions such as whether the character is different enough from existing ones, whether it has the interesting appearance, is it pleasant for observing. The second aspect is related to technical requirements. In this case, there are questions such as: is a silhouette of the character stands out enough form the background or environment, whether a person can in every moment recognize the character and follow them (Solan, 2015b).

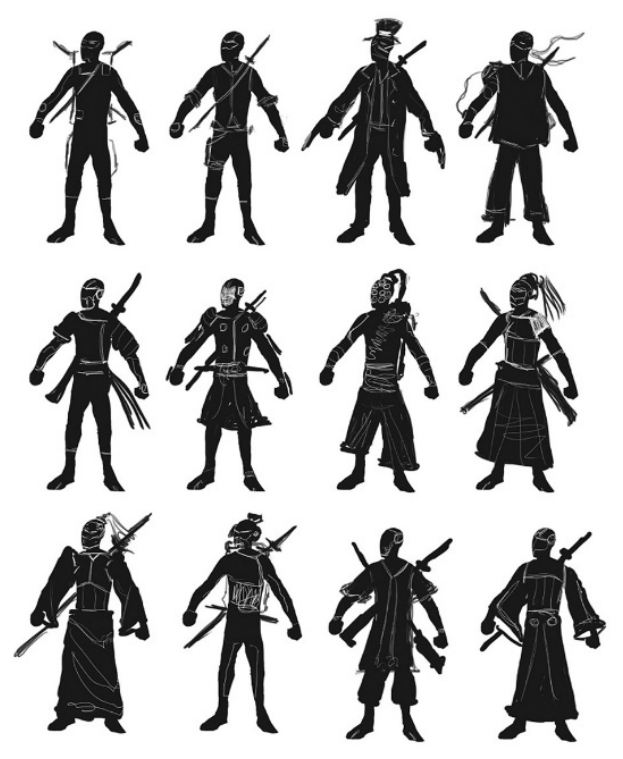

Figure 2: Silhouette of the character (Solan, 2015)

It is recommended to think about the functionality of the character during the silhouette phase. Regardless of the fictional nature of the character $r$, they have to be functional. This also refers to the design of the body of the character - if the character is a monster with wings, the wings need to be strong enough to carry the monster safely. All additional parts and items on the character have to be in real relationship with the character - to convey a sense of real functional use.

\subsection{Colour - analysis and importance of the colour in the process of the character design}

The aim of the better representation of the character is to be easily understood by the observer and to fit in their mental models which are common to all people. In order to accomplish that, it is necessary to analyse and research colour, its use, and symbolism. It is known that the observer perceives the world and environment around mainly thanks to colour. During the time, different colours take the role of certain symbols which become widely accepted and established in mental models of people.

With the symbolism of the colours as guidelines, it is possible to connect colours with the personality traits of the character. In the visual culture, the most appropriate element for conveying the sense of good or bad is the colour. Characters in the animated movies of comic books have opposed appearance which is conveyed throughout colour. For example, good character opposed to bad character or hero opposed to a villain is presented with opposed colours in the colour spectrum. The most used opposed colour pairs are black - white and red - blue. The first pair, black - white, represents evil against good or negative character versus positive. Additionally, black colour represents a shadow character which cannot be presented as an evil character. The second colour pair, red - blue, is commonly used for the hero and villain design. The red colour is often used for villains, whereas blue is used for the appearance of the hero. When designing hero characters, for costume design are usually consisted of primary colours - red, blue, yellow or gold whereas costumes for villains are usually designed utilizing secondary colours - green, purple and grey. The most known example for this is the Supermen costume (which is a combination of the blue and red) and costume of his main opponent Jocker, from the Batman saga, (who wears a combination of purple and green costume) (Tvtropes, 2017). 


\section{CHARACTER DESIGN CONCEPT}

After the analysis of the most important influential factors on the character design process, the important phase to mention is character design concept phase. This phase is always at the beginning of the character design process. It is important to know that the previously described elements are defined in this phase. This is the first phase in the production pipeline and it is closely connected to the scenario and detailed textual description of the character. Archetypes, facial expression, look, shape and body posture are the usually defined in the game scenario where witter or scenarist create an initial idea of the character appearance. The main task for the concept artist is to make a visual representation of the character described in the written scenario (Figure 3). In the concept phase, it is recommended to define character precisely with all details and personality characteristic, with combining their inner and external characteristics (Vaughan, 2012).

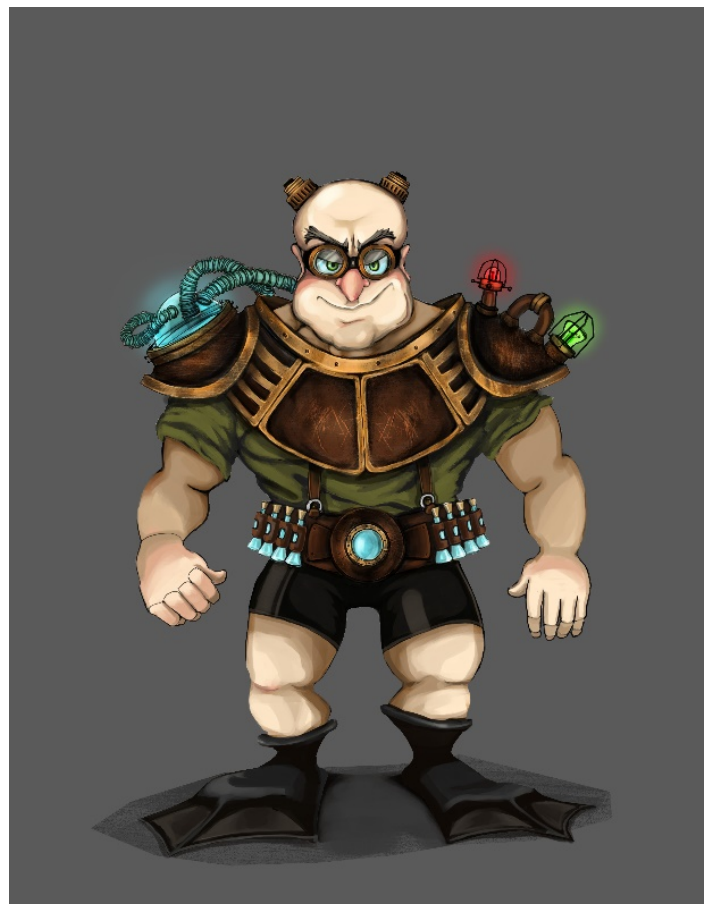

Figure 3: Concept of the character based on the scenario

\section{CONCLUSION}

Character design process is crucial segment because characters lead observer throughout the story. The primary goal of the character design process is a connection with the audience. Characters are an inevitable part of everyday life, they are present in every aspect of life both as virtual characters and physical characters. People commonly identify themselves with the characters, which is natural. Characters represent personalities which are in constant interaction with the people. Character designers have the power to define the type of the character for people to identify, as well as the target group for their product. The importance of the character can be seen from the definition of fun because characters are an integral part of the entertainment industry. "Fun represents the process which involves exploration of the relationships throughout simulations which enable persona to identify themselves with substitute persona (for example avatar) and in that way create subjective experience and relationships" (Solan, 2015b). Designers of the characters have to know precisely which part of the character has to be emphasized in order to create a recognizable character which is receptive for identification. Besides the detailed analysis of the character traits, the analysis of the references is required. Collecting and observing and analysing references is essential for finding answers to questions such as: "What part of the design is crucial for an appearance like this?" or "Why is the character good or bed" or "Why is this character adored by the people?". Constant search and giving answers to these questions lead to successful character design. 


\section{ACKNOWLEDGMENTS}

This work was supported by the Serbian Ministry of Science and Technological Development, Grant No.: 35027 "The development of software model for improvement of knowledge and production in graphic arts industry."

\section{REFERENCES}

[1] Tillman, B.: "Creative Character Design", (Focal Press, Massachusetts, 2011a), page 5.

[2] Tillman, B.: "Creative Character Design", (Focal Press, Massachusetts, 2011b), Chapter 2.

[3] Isbister, K.: "Better game characters by design a psychological approach", (Morgan Kaufmann publications, San Francisco, 2006a), Chapter 5.

[4] Isbister, K.: "Better game characters by design a psychological approach", (Morgan Kaufmann publications, San Francisco, 2006b), page 145.

[5] Isbister, K.: "Better game characters by design a psychological approach", (Morgan Kaufmann publications, San Francisco, 2006c), Chapter 6.

[6] Solan, R. J.S.: "Virtual character design for games and interactive media", (Taylor \& Francis Group, Boca Raton, 2015a), page 26.

[7] Solan, R. J.S.: "Virtual character design for games and interactive media", (Taylor \& Francis Group, Boca Raton, 2015b), page 30.

[8] Tvtropes, Good Colors, Evil Colors, Tvtropes, URL: https://tvtropes.org/pmwiki/pmwiki.php/Main/GoodColorsEvilColors (last request: 2018-10-05)

[9] Vaughan, W.: "Digital modeling", (New Riders, 2012), Chapter 2.

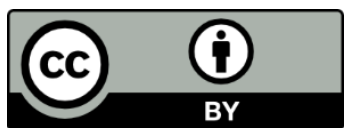

(C) 2018 Authors. Published by the University of Novi Sad, Faculty of Technical Sciences, Department of Graphic Engineering and Design. This article is an open access article distributed under the terms and conditions of the Creative Commons Attribution license 3.0 Serbia (http://creativecommons.org/licenses/by/3.0/rs/). 